\title{
Bevacizumab Added to Neoadjuvant Chemotherapy in HER2-Negative Non-Metastatic Breast Cancer
}

\author{
Guoxing Wan ${ }^{\bowtie}$, Fengjun Cao, Xuanbin Wang, Xue Sun \\ Department of Oncology, Renmin Hospital, Hubei University of Medicine, Shiyan 442000, China. \\ $\triangle$ Corresponding author: Guoxing Wan, MD, PhD, Department of Oncology, Renmin Hospital, Hubei University of Medicine, 39 Chaoyang Road, 442000 \\ Shiyan, China. Phone: +86-719-8637385; Fax: +86-719-8637385; Email: 15gxwan@stu.edu.cn \\ (C) Ivyspring International Publisher. This is an open access article distributed under the terms of the Creative Commons Attribution (CC BY-NC) license \\ (https://creativecommons.org/licenses/by-nc/4.0/). See http://ivyspring.com/terms for full terms and conditions.
}

Received: 2018.08.25; Accepted: 2018.11.01; Published: 2019.01.01

\section{To the Editor,}

Recombinant humanized monoclonal antibody bevacizumab binding and inactivating VEGF-A has recently been a particularly attractive focus of research in anti-angiogenic treatment strategy for breast cancer especially the HER-2 negative subtype [1]. Three recently published phase III randomized controlled trials-ARTemis [2], GBG 44- GeparQuinto [3] and SWOG S0800 trial [4]-have showed that adding bevacizumab to neoadjuvant chemotherapy (NAC) increased the pathological complete response (pCR) but did not favor the survival of HER-2 negative non-metastatic breast cancer (NMBC), while NSABP B-40 trial [5] contradicted the findings by demonstrating an improved survival particularly disease-free survival (DFS). To settle the disputes, we therefore performed a meta-analysis to evaluate the survival benefit and risk of this treatment strategy.

Involving 4122 participants with a median follow-up from 3 to 4.7 year, the pooled data suggested no significant effect of bevacizumab on either DFS or overall survival (OS). Similar results on DFS were also found according to the hormone receptor (HR) status. Unexpectedly, a significantly reduced DFS was indicated in patients achieving a pCR (hazard ratio, 2.36; 95\% CI, 1.33-4.19) while not in patients without a pCR. The stratification analyses by HR status regarding OS showed no significant effect as well (Figure 1). Involving two trials with a total of 1897 participants, the result showed significantly increased risk of any surgical complications (risk ratio, 1.39; 95\% CI, 1.20-1.62) in patients receiving NAC and neoadjuvant bevacizumab (Figure 1).

Although an individual patient-level metaanalysis would be ideal, our results highlighted that adding bevacizumab to NAC for HER-2 negative NMBC did not provide survival benefit but significantly increased the risk of surgical complications. Also, the results did not support the role of HR status in discriminating the effect of bevacizumab, which contradicted the finding from NSABP-B40 that addition of bevacizumab resulted in improved survival especially in HR-positive patients. In NSABP B40 but not in other trials, patients received bevacizumab not just preoperatively but also postoperatively, such substantial differences may have contributed to the discordant results because bevacizumab was able to affect not only the primary tumor but also dormant micrometastases. Although most previous trials incorporating the currently included trials showed consistently an increased PCR rate with neoadjuvant bevacizumab, reduced DFS was revealed in patients achieving $\mathrm{PCR}$ in the present study, suggesting that the PCR advantage seemed not always to be translated into a survival advantage. Our analysis failed to demonstrate this benefit while confirmed increased toxicity, supporting utmost cautions against the adoption of neoadjuvant bevacizumab in this setting.

\section{Acknowledgements}

GXW contributed to the initial concept, protocol writing, data interpretation and manuscript writing; FJC contributed to data collection and interpretation, manuscript review; XBW and XS helped with protocol writing, data collection and interpretation.

\section{Competing Interests}

The authors have declared that no competing interest exists. 


\begin{tabular}{|c|c|c|c|c|}
\hline Characteristics & ARTemis[2] & NSABP B-40[5] & GBG 44-GeparQuinto[3] & SWOG0800[4] \\
\hline Clinicaltrial number & NCT01093235 & NCT00408408 & NCT 00567554 & NCT00856492 \\
\hline Design & phase III, RCT, open label & phase III, RCT, open label & phase III, RCT, open label & phase III, RCT, open label \\
\hline Country & UK & Canada and USA & USA & USA \\
\hline Baseline NAC & T-FEC & $\begin{array}{l}T \rightarrow A C \text { or } T X \rightarrow A C \\
\text { or } T G \rightarrow A C\end{array}$ & $\mathrm{EC} \rightarrow \mathrm{T}$ & Nab-paclitaxel $\rightarrow$ AC \\
\hline Setting & $T \geqslant 2 \mathrm{~cm}, \operatorname{Node}( \pm)$ & T1c-T3,NO-N2a & T1-4,Node(+) & Locally advanced/inflammatory \\
\hline Bev dose(cycles) & $15 \mathrm{mg} / \mathrm{kg}$ iv,q3w(×4) & $15 \mathrm{mg} / \mathrm{kg}$ iv,q3w(×6) & $15 \mathrm{mg} / \mathrm{kg}$ iv,q3w(×8) & $10 \mathrm{mg} / \mathrm{kg}$ iv,q2w(×6) \\
\hline Postoperative use of Bev & no & yes & no & no \\
\hline Median follow-up & 3.5 years & 4.7 years & 3.8 years & 3 years \\
\hline No. of Patients(Bev vs. no Bev) & 399 vs. 401 & 592 vs. 594 & 956 vs. 969 & 98 vs. 113 \\
\hline Age & $\begin{array}{l}\leqslant 50 \text { years }(68 \%) \\
>50 \text { years }(32 \%)\end{array}$ & $\begin{array}{l}<50 \text { years }(52 \%) \\
\geqslant 50 \text { years }(48 \%)\end{array}$ & $\begin{array}{l}<40 \text { years }(16 \%) \\
\geqslant 40 \text { years }(84 \%)\end{array}$ & Median 51.5 (22-75) years \\
\hline Hazard ratio $(95 \% \mathrm{Cl})$ for Bev & $1.18(0.89-1.57)$ & $0.80(0.63-1.01)$ & $1.03(0.84-1.25)$ & $0.89(0.48-1.65)$ \\
\hline$P$ value for DFS & 0.25 & 0.06 & 0.784 & 0.71 \\
\hline Hazard ratio $(95 \% \mathrm{Cl})$ for Bev & $1.26(0.90-1.76)$ & $0.65(0.49-0.88)$ & $0.97(0.75-1.26)$ & $0.84(0.41-1.73)$ \\
\hline$P$ value for OS & 0.19 & 0.004 & 0.842 & 0.64 \\
\hline Any surgical complications(\%) & - & $40.0 \%$ vs. $28.6 \%$ & $14.7 \%$ vs. $10.9 \%$ & - \\
\hline
\end{tabular}

\section{Pooled analysis}

\begin{tabular}{cccc}
\hline Outcome & $\begin{array}{c}\text { No. of participants } \\
\text { (No. of trials) }\end{array}$ & $\begin{array}{c}\text { Hazard Ratio or } \\
\text { Risk Ratio(95\%Cl) }\end{array}$ & $\begin{array}{c}\text { Heterogeneity } \\
\text { test }\end{array}$ \\
\hline Disease-free survival & $4120(4$ trials) & $0.98(0.85-1.11)$ & $\mathrm{P}=0.19, \mathrm{I}^{2}=37 \%, \mathrm{~F}$ \\
pCR(yes) & $449(2$ trials) & $2.36(1.33-4.19)$ & $\mathrm{P}=0.51, \mathrm{I}^{2}=0 \%, \mathrm{~F}$ \\
PCR(no) & $2153(2$ trials) & $0.98(0.82-1.17)$ & $\mathrm{P}=0.88, \mathrm{I}^{2}=0 \%, \mathrm{~F}$ \\
HR(+) & $2112(3$ trials) & $1.03(0.66-1.60)$ & $\mathrm{P}=0.04, \mathrm{I}^{2}=69 \%, \mathrm{R}$ \\
HR(-) & $1208(3$ trials) & $0.93(0.76-1.15)$ & $\mathrm{P}=0.23, \mathrm{I}^{2}=33 \%, \mathrm{~F}$ \\
Overall survival & $4122(4$ trails) & $0.91(0.67-1.23)$ & $\mathrm{P}=0.03, \mathrm{I}^{2}=68 \%, \mathrm{R}$ \\
HR(+) & $851(2$ trials) & $0.94(0.34-2.59)$ & $\mathrm{P}=0.09, \mathrm{I}^{2}=66 \%, \mathrm{R}$ \\
HR(-) & $546(2$ trials) & $0.74(0.52-1.05)$ & $\mathrm{P}=0.36, \mathrm{I}^{2}=0 \%, \mathrm{~F}$ \\
Surgical complications & $1897(2$ trials) & $1.39(1.20-1.62)$ & $\mathrm{P}=0.87, \mathrm{I}^{2}=0 \%, \mathrm{~F}$ \\
\hline
\end{tabular}

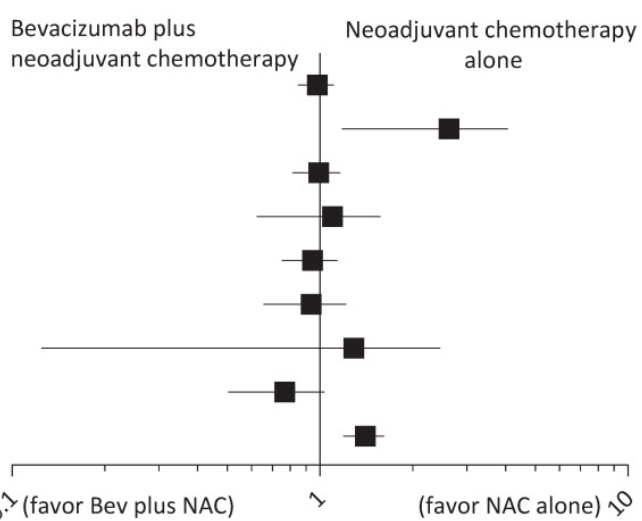

Figure 1. Survival and surgical complications for bevacizumab plus neoadjuvant chemotherapy versus neoadjuvant chemotherapy alone. Bev, bevacizumab; NAC, neoadjuvant chemotherapy; RCT, randomized controlled trial; T, docetaxel; FEC, fluorouracil plus epirubicin plus cyclophosphamide; AC, doxorubicin plus cyclophosphamide; TX, docetaxel plus capecitabine; TG, docetaxel plus gemcitabine; EC, epirubicin plus cyclophosphamide; q3w, every third week; iv, intravenously; DFS, disease-free survival; OS, overall survival; PCR, pathological complete response; HR, hormone receptor*A hazard ratio or risk ratio less than 1 favors the outcome by Bev plus NAC, while a hazard ratio or risk ratio greater than 1 favors the outcome by NAC alone. F, fixed-effect model; R, random-effect model.

\section{References}

1. Kumler I, Christiansen OG, Nielsen DL. A systematic review of bevacizumab efficacy in breast cancer. Cancer Treat Rev 2014; 40(8): 960-73.

2. Earl HM, Hiller L, Dunn JA et al. Disease-free and overall survival at 3.5 years for neoadjuvant bevacizumab added to docetaxel followed by fluorouracil, epirubicin and cyclophosphamide, for women with HER2 negative early breast cancer: ARTemis Trial. Ann Oncol 2017; 28(8): 1817- 24.

3. von Minckwitz G, Loibl S, Untch $M$ et al. Survival after neoadjuvant chemotherapy with or without bevacizumab or everolimus for HER2-negative primary breast cancer (GBG 44-GeparQuinto) dagger. Ann Oncol 2014; 25(12): 2363-72.

4. Nahleh ZA, Barlow WE, Hayes DF et al. SWOG S0800 (NCI CDR0000636131): addition of bevacizumab to neoadjuvant nab-paclitaxel with dose-dense doxorubicin and cyclophosphamide improves pathologic complete response $(\mathrm{pCR})$ rates in inflammatory or locally advanced breast cancer. Breast Cancer Res Treat 2016; 158(3): 485-95.

5. Bear HD, Tang G, Rastogi $P$ et al. Neoadjuvant plus adjuvant bevacizumab in early breast cancer (NSABP B-40 [NRG Oncology]): secondary outcomes of a phase 3, randomised controlled trial. Lancet Oncol 2015; 16(9): 1037-48. 